\title{
Hydrazide-Tandem Mass Tag
}

National Cancer Institute

\section{Source}

National Cancer Institute. Hydrazide-Tandem Mass Tag. NCI Thesaurus. Code C161875.

A tandem mass tag reagent preparation that is coupled to a carbonyl-reactive hydrazide group that enables labeling and quantitative profiling of glycopeptides, carbohydrates, steroids, or oxidized proteins. 